\title{
Microscopic spermatic cord denervation for chronic orchialgia/ chronic scrotal content pain: operative outcomes and predictors of failure
}

\author{
Prithvi B. Murthy, Neel V. Parekh, Sarah C. Vij, Daniel A. Shoskes \\ Department of Urology, Glickman Urological \& Kidney Institute, Cleveland Clinic, Cleveland, OH, USA \\ Contributions: (I) Conception and design: All authors; (II) Administrative support: SC Vij, DA Shoskes; (III) Provision of study materials or patients: \\ SC Vij, DA Shoskes; (IV) Collection and assembly of data: All authors; (V) Data analysis and interpretation: All authors; (VI) Manuscript writing: All \\ authors; (VII) Final approval of manuscript: All authors. \\ Correspondence to: Prithvi B. Murthy, MD. Glickman Urological \& Kidney Institute, Cleveland Clinic, 9500 Euclid Avenue, Cleveland, OH 44195, \\ USA. Email: murthyp@ccf.org.
}

\begin{abstract}
Background: To describe our institutional outcomes with microscopic spermatic cord denervation (MSCD) for chronic scrotal content paint (CSCP) and identify predictors of treatment failure.

Methods: Retrospective chart review was performed to identify all MSCD performed by two surgeons at a single institution from 2010-2019. Patient demographic data and operative outcomes were collected. Patients were excluded from analysis if no post-operative follow up was available. Success was defined as complete resolution of bothersome pain. Multivariable logistic regression was utilized to identify predictors of treatment failure.

Results: During the study period, 101 patients were identified in which 113 MSCD procedures were performed. Final analysis included 103 procedures across 93 patients. Mean age was 41.8 years (SD 13.2), mean BMI was $29.2 \mathrm{~kg} / \mathrm{m}^{2}$ (SD 5.96) and median months of pain preceding surgery were 24 (range, 3-300 months). Overall, 75/103 (73\%) MSCD were successful. Of the failures, 5 patients had recurrence of pain greater than 6 months after surgery. Only the presence of pelvic floor muscle spasm (PFMS) independently predicted MSCD failure (OR 3.95, P=0.02). 9 of 19 (47\%) patients with PFMS experienced treatment failure, while 19 of 84 (23\%) without PFMS experienced failure.

Conclusions: MSCD offers a therapeutic option for patients with refractory CSCP. The presence of PFMS is associated with lower surgical success rates. Patients with pre-operatively identified PFMS should be counseled regarding a higher risk of treatment failure.
\end{abstract}

Keywords: Spermatic cord; denervation; orchialgia; pelvic floor

Submitted Feb 12, 2020. Accepted for publication Aug 04, 2020.

doi: $10.21037 /$ tau-20-561

View this article at: http://dx.doi.org/10.21037/tau-20-561

\section{Introduction}

Chronic orchialgia (CO) and the more broadly encompassing term chronic scrotal content pain (CSCP) may pose a diagnostic and therapeutic challenge. In patients without a defined etiology, microscopic spermatic cord denervation can provide long-term relief when primary conservative measures fail (1). While Benson et al. demonstrated that pain improvement after spermatic cord block is associated with symptom relief after MSCD, this is already routine practice, and further literature identifying indicators of success after MSCD is limited (2). Since the initial description of MSCD in a series of two patients by Devine and Schellhammer in 1978, larger contemporary MSCD series have demonstrated success rates ranging from $71-96 \%(3-8)$.

More recently, Parekattil et al. have identified an 
anatomic basis for successful MSCD in describing the trifecta nerve complex, a group of small diameter $(<1 \mathrm{~mm})$ nerves demonstrating Wallerian degeneration in the cremasteric muscle fibers, peri-vasal sheath, and posterior spermatic cord lipomatous tissues (9). Kavoussi has externally validated these findings, demonstrating that a targeted MSCD limited to the trifecta nerve complex is as efficacious as a full skeletonization of the spermatic cord (10). Since there may not be a clear anatomic correlate, pain outside the scrotum and depressive symptoms have been shown to be associated with failure of MSCD (7). In addition, as nearly $58 \%$ of patients with chronic prostatitis (CP)/chronic pelvic pain syndromes (CPPS) report a component of testicular pain, it is critical to categorize patients in which MSCD may be unsuccessful (11). We hypothesize that the presence of pelvic floor muscle spasm, as a primary extra-scrotal pathology, or prior inguinoscrotal surgery may predict for treatment failure after MSCD. In the current study, we report our single institution outcomes with MSCD and identify predictors of treatment failure. We present the following article in accordance with the STROBE reporting checklist (available at http://dx.doi. org/10.21037/tau-20-561).

\section{Methods}

We performed a retrospective chart review of all patients undergoing MSCD by two surgeons at a single institution from January of 2010 to June of 2019. All procedures performed in this study were in accordance with the Declaration of Helsinki (as revised in 2013) and approved by the Institutional Review Board (IRB 06-097). Because of the retrospective nature of the research, the requirement for informed consent was waived. Both surgeons performed a more traditional microscopic sub-inguinal denervation which included the trifecta nerve-complex. This consisted of division of spermatic fascia including cremasteric fibers, division or stripping of the peri-vasal sheath, ligation of several spermatic veins (sparing at least one), dividing the peri-spermatic cord and posterior lipomatous tissues with preservation of all arteries and at least one lymphatic. Patient history and physical exam findings as well as demographics, medical and surgical history related to the patient's orchialgia were collected. The patient history also focused on extra-testicular pain, and pelvic floor muscle spasm was diagnosed via history with confirmatory digital rectal exam (DRE). Patients with pelvic floor muscle spasm were offered pelvic floor physical therapy with a specialized pelvic floor physiotherapist, though not required to undergo physical therapy prior to MSCD. If patients had isolated scrotal content pain on external genital exam and did not mention pain outside the scrotum on history, they did not necessarily undergo pre-operative DRE to evaluate for pelvic floor muscle spasm. Patients had been previously managed with a variety of conservative management strategies, most commonly at the discretion of the referring physician, which may have included non-narcotic analgesics, antibiotics, and specialized pain management with local or regional nerve blocks. The primary outcome of interest was complete resolution of bothersome pain as defined by the patient. Pain was quantified with the Chronic Orchialgia Symptom (COSI) Index following its validation in 2018, and a patient reported $1-10$ pain scale prior. All patients pre-operatively underwent a spermatic cord block consisting of $10 \mathrm{cc}$ of an equal mixture of $0.25 \%$ bupivacaine and $1 \%$ lidocaine. Patients were only offered MSCD if they had elimination or near-elimination of all self-defined bothersome pain with the spermatic cord block. In addition, they were counseled that MSCD would not alleviate pain to any degree greater than the maximum effect achieved by the spermatic cord block. We counsel patients in this manner based on historic, patient reported results. Given the geographical referral pattern of our patient population, we did not pre-define a follow up to note pain resolution. Patients are asked to follow up as needed and are offered office based visits, telephone based encounters or virtual visits as options. Patients were included for analysis if they had at least one post-operative telephone encounter, virtual visit, or office visit to discuss pain resolution. They were excluded if there was no follow up. Any recurrence of pain at any time point following surgery was considered treatment failure. DRE for pelvic floor muscle spasm was performed in patients with persistent CSCP after MSCD.

Statistical analysis was performed using SPSS (IBM, Armonk, NY). We identified potential predictors of treatment failure with pre-defined continuous and categorical variables based on clinical relevance, after which multivariable logistic regression was performed. $\mathrm{P}$ values $<0.05$ were considered statistically significant. Continuous variables were described as means or medians along with standard deviation (SD) or first and third quartile, respectively.

\section{Results}

In 101 patients identified during the study period, 113 
Table 1 Patient demographics

\begin{tabular}{lc}
\hline Demographics & Value \\
\hline No. patients & 92 \\
No. surgeries included & 103 \\
Mean age (years, SD; range) & $41.8(13.2 ; 20-79)$ \\
Laterality, n (\%) & $47(46 \%)$ \\
Right & $22(22 \%)$ \\
Prior vasectomy, n (\%) & $9(8.8 \%)$ \\
Prior epididymectomy, $\mathrm{n}(\%)$ & $50(49 \%)$ \\
Other prior inguinal/scrotal surgery, $\mathrm{n}(\%)$ & $19(19 \%)$ \\
Pelvic floor muscle spasm, $\mathrm{n}(\%)$ & $24(12-60)$ \\
Median time with orchialgia (months, & \\
Q1-Q3) & $28.4(6.0 ; 18.1-47.1)$ \\
Mean BMI (kg/m², SD; range) & $75(73 \%)$ \\
\hline Resolution of pain, $\mathrm{n}$ (\%) &
\end{tabular}

MSCD procedures were performed. Bilateral surgery was performed in 12 patients, either as a staged operation early in our experience, or currently as a concomitant procedure. Ten MSCD were excluded from analysis due to lack of follow up, so the final cohort consisted of 103 MSCD across 93 patients. Median follow up time was 3.1 months (Q1 1.5, Q3 11.6, range, 0.3-74.3). Patient demographics and relevant clinical history are displayed in Table 1. Mean age was 41.8 years (SD 13.2, range, 20-79), mean BMI was $28.4 \mathrm{~kg} / \mathrm{m}^{2}$ (SD 6.0, range, 18.1-47.1) and median months of pain preceding surgery were 24 (Q1 12, Q3 60, range, 3-300 months). 22 patients (22\%) had previously underwent vasectomy and 19 patients (19\%) had pelvic floor muscle spasm (PFMS) based on physical examination. 50 patients $(49 \%)$ had prior ipsilateral hernia, groin or scrotal surgery which included orchidopexy and hydrocelectomy. Overall 75/103 (73\%) MSCD were successful.

Odds ratios (OR) for predicting MSCD failure are shown in Table 2. PFMS independently predicted MSCD failure, with an OR of 3.95. 9 of 19 (47\%) patients with PFMS experienced treatment failure while 19 of 84 (23\%) without PFMS experienced failure. When excluding patients with PFMS, the overall success rate was $77 \%$ (65/84). None of the other co-variates were independently associated with failure of MSCD. 15 of 22 (68\%) and 37 of 50 (74\%) patients with history of vasectomy and prior inguinal or scrotal surgery, respectively, had treatment success.
Of the 5 patients experiencing recurrence of pain after 6 months, one had pre-existing PFMS. Two patients that had initial treatment failure noted significant improvement after genitofemoral nerve block, one of which went on to receive genitofemoral nerve cryotherapy. Another patient underwent cryotherapy of the spermatic cord with post-therapy COSI of 11 from 25 pre-therapy (12). One additional patient had sub-inguinal orchiectomy due to pain. Complications of surgery were as follows: one patient had persistent pain with scrotal Doppler demonstrating no flow and underwent sub-inguinal orchiectomy, two patients had post-operative hydroceles of which one resolved on its own and another required operative repair, and four patients had superficial wound infections that were managed with antibiotics and local wound care. Of the 9 patients with PFMS and MSCD failure, only two followed through with pelvic floor physical therapy. The patient undergoing therapy at our institution reported moderate resolution in his CSCP, and the patient having therapy at an outside facility did not.

\section{Discussion}

MSCD is an effective treatment for chronic scrotal content pain. Since its first description in two patients by Devine and Schellhammer in 1978, multiple studies, including a prospective multi-center trial by Marconi et al., have demonstrated success rates ranging from $71-96 \%(3-5,12)$. Overall, we report a $78 \%$ MSCD success rate $(75 / 103)$ in a diverse cohort of men, and noted that PFMS is independently associated with treatment failure of MSCD.

Research on the anatomic basis of MSCD by Parekattil et al. led to the description of the "trifecta nerve complex" in patients with CSCP (9). This group of small diameter $(<1 \mathrm{~mm})$ nerves located in the cremasteric muscle fibers, peri-vasal sheath, and posterior spermatic cord lipomatous tissues were noted to have a significantly increased rate of Wallerian degeneration when compared to control patients; this pattern of nerve degeneration has been associated with the development of neuropathic pain (13-15). By staining the pan-neuronal marker $P G P$ 9.5, sympathetic nerve marker tyrosine bydroxylase, and sensory nociceptive marker calcitonin gene-related peptide, Oka et al. similarly identified a high concentration of sensory and sympathetic nerve fibers localized to the vas deferens and spermatic fascia (16). Following this anatomic roadmap, Calixte $e t a l$. demonstrated a greater than $50 \%$ resolution of pain in $83 \%$ 
Table 2 Predictors of MSCD failure

\begin{tabular}{|c|c|c|c|}
\hline & Odds ratio & $95 \% \mathrm{Cl}$ & $P$ value \\
\hline BMI (per kg/m²) & 0.96 & $0.91-1.06$ & 0.70 \\
\hline Months of Pain (per month) & 1.00 & $0.99-1.00$ & 0.48 \\
\hline Side (R vs. L) & 1.18 & $0.46-3.00$ & 0.74 \\
\hline Prior epididymectomy & 0.60 & $0.09-3.90$ & 0.60 \\
\hline Other prior inguinal/scrotal surgery & 0.99 & $0.38-2.66$ & 0.99 \\
\hline Presence of pelvic floor muscle spasm & 3.95 & $1.27-12.26$ & 0.02 \\
\hline
\end{tabular}

of men of a cohort of 860 patients undergoing a roboticassisted MSCD targeting only the nerve complex, sparing all arteries, veins, lymphatics and the vas deferens (6).

If a source dependent, anatomically driven denervation serves as the basis for pain resolution in CO/CSCP, it stands to reason that referred pain from PFMS would preclude a successful MSCD. In our cohort, we found that the presence of PFMS predicted MSCD failure with an OR of 3.95. Shiraishi et al. similarly noted that the presence of pain outside of the scrotum, such as the perineal, lower abdominal and femoral regions, independently predicted for treatment failure when performing a high-inguinal approach for MSCD in CSCP (OR 15.3, CI: 1.0-22.9, $\mathrm{P}=0.048$ ) (7). Referred scrotal pain in PFMS may be due to nociceptor activating inflammatory cytokines at the level of the pelvic musculature, which is not targeted by MSCD $(17,18)$. Moreover, significant overlap exists between patients with pelvic pain and CSCP. Wagenlehner et al. noted that testicular pain was present in $58 \%$ of patients in a multinational cohort of 1563 patients with CP/CPPS, and was second only to perineal pain $(63 \%)$ with regards to location (11). When examining the association between CSCP and pelvic floor overactivity, Planken et al. identified that $93 \%$ of patients with CSCP evaluated at their Pelvic Floor Department had at least 1 symptom suspicious of pelvic floor dysfunction, which included the domains of micturition, defecation and sexual function; $49 \%$ of patients had symptoms in all three domains (19). Therefore, it is necessary to elicit a history relating to pelvic floor muscle spasm for each patient presenting with CO, and perform DRE as the history dictates.

Furthermore, pelvic floor therapy can be offered as an alternative to MSCD in cases of concomitant CSCP and
PFMS. In addition, patients undergoing MSCD with PFMS should be counseled on the potential for higher surgical failure rates. Shoskes et al. have previously demonstrated that pelvic floor physical therapy (PFPT) is an efficacious treatment for pelvic floor tenderness in patients with $\mathrm{CP} /$ CPPS (20). With regards to the CSCP population, Farrell et al. performed PFPT in a cohort of 30 men with CSCP and pelvic floor tightness (21). They noted that $44 \%$ of patients reported none or minor residual pain after an average of 12 PFPT sessions. PFMS has also been successfully treated with trigger point injections and dry needling techniques (18). In our cohort of 9 men with PFMS that failed MSCD, only two elected to trial PFPT, with moderate resolution of CSCP in the patient undergoing therapy at our institution.

We did not identify any other demographic or clinical factor associated with treatment failure, such as age, duration of symptoms or history of prior inguinal or scrotal surgery, particularly vasectomy and epididymectomy. Similarly, Shoskes et al. did not identify these clinical parameters to individually significantly impact the total score in their multi-institutional validation study of the Chronic Orchialgia Symptom Index, suggesting that one clinical or demographic factor does not bias degree of pain or treatment response (12). Tan et al. performed MSCD on 28 testicular units for the post-vasectomy pain syndrome (PVPS) and reported an overall success rate of $71 \%$; they did not find that their success rates were influenced by a prior procedure for PVPS, such as vasovasostomy (22). In addition, Larsen et al. have shown that MSCD success rates are similar between men who have and have not had prior attempts to surgically correct CSCP, such as epididymectomy, varicocelectomy or orchidopexy (66\% vs. 
$75 \%, \mathrm{P}=0.4)(23)$.

The retrospective nature of our study limited our ability to quantify pre and post-operative pain with the validated Chronic Orchialgia Symptom Index. However, while much of the MSCD literature uses the Visual Analog Scale to characterize pain in CO/CSCP, the VAS is not validated for CSCP. Though use of the COSI would be ideal, it was validated in 2018 and not available for the majority of our patients. We were also unable to establish routine minimum follow up times in our patient cohort which may limit identification of late failures. Our practice is to allow or patients to follow up as needed, which includes tele-health based visits. Also, we did not routinely perform DRE on patients with isolated scrotal content pain, which may have led to the under detection of PFMS. Given this, our diagnosis of PFMS in those that failed MSCD may have been confounded by new-onset PFMS after MSCD. However, this decision was based on a focused patient history that includes questions relating to voiding and sexual function, which are almost invariably associated with pelvic floor dysfunction (19). When the history suggested an extra-scrotal source of pain, a DRE was performed. Considering these limitations, we still believe that our findings apply to men with CSCP and PFMS given our patient-oriented definition of treatment success, accessible follow-up options, and low threshold in performing DRE if an extra-scrotal etiology for pain was suspected.

\section{Conclusions}

Patients with pelvic floor muscle spasm are more likely to experience treatment failure following microscopic subinguinal spermatic cord denervation for chronic scrotal content pain, even with a favorable response to spermatic cord block. A history relating to pelvic floor muscle spasm should be taken for all patients presenting with chronic orchialgia or chronic scrotal content pain, and digital rectal exam should be performed if the history is suggestive. If underlying pelvic floor dysfunction exists, pelvic floor physical therapy can be offered to patients prior to spermatic cord denervation. History of prior vasectomy, epididymectomy, prior inguinal or scrotal surgery or other patient demographic factors were not associated with treatment failure.

\section{Acknowledgments}

Funding: None.

\section{Footnote}

Reporting Checklist: The authors have completed the STROBE reporting checklist. Available at http://dx.doi. org/10.21037/tau-20-561

Data Sharing Statement: Available at http://dx.doi. org/10.21037/tau-20-561

Conflicts of Interest: All authors have completed the ICMJE uniform disclosure form (available at http://dx.doi. org/10.21037/tau-20-561). DAS serves as an unpaid editorial board member of Translational Andrology and Urology from Aug 2020 - Jul 2022; and he reports other from Triurol, personal fees from UroGen, outside the submitted work. The authors have no other conflicts of interest to declare.

Ethical Statement: The authors are accountable for all aspects of the work in ensuring that questions related to the accuracy or integrity of any part of the work are appropriately investigated and resolved. All procedures performed in this study were in accordance with the Declaration of Helsinki (as revised in 2013) and approved by the Institutional Review Board (IRB 06-097). Because of the retrospective nature of the research, the requirement for informed consent was waived.

Open Access Statement: This is an Open Access article distributed in accordance with the Creative Commons Attribution-NonCommercial-NoDerivs 4.0 International License (CC BY-NC-ND 4.0), which permits the noncommercial replication and distribution of the article with the strict proviso that no changes or edits are made and the original work is properly cited (including links to both the formal publication through the relevant DOI and the license). See: https://creativecommons.org/licenses/by-nc-nd/4.0/.

\section{References}

1. Sigalos JT, Pastuszak AW. Chronic orchialgia: epidemiology, diagnosis and evaluation. Transl Androl Urol 2017;6:S37-43.

2. Benson JS, Abern MR, Larsen S, et al. Does a Positive Response to Spermatic Cord Block Predict Response to Microdenervation of the Spermatic Cord for Chronic Scrotal Content Pain? J Sex Med 2013;10:876-82.

3. Strom KH, Levine LA. Microsurgical Denervation of the Spermatic Cord for Chronic Orchialgia: Long-Term 
Results From a Single Center. J Urol 2008;180:949-53.

4. Devine CJ, Schellhammer PF. The use of microsurgical denervation of the spermatic cord for orchialgia. Trans Am Assoc Genitourin Surg 1978;70:149-51.

5. Marconi M, Palma C, Troncoso P, et al. Microsurgical Spermatic Cord Denervation as a Treatment for Chronic Scrotal Content Pain: A Multicenter Open Label Trial. J Urol 2015;194:1323-7.

6. Calixte N, Tojuola B, Kartal I, et al. Targeted Robotic Assisted Microsurgical Denervation of the Spermatic Cord for the Treatment of Chronic Orchialgia or Groin Pain: A Single Center, Large Series Review. J Urol 2018;199:1015-22.

7. Shiraishi K, Tabara M, Matsuyama H. High Inguinal Microsurgical Denervation of the Spermatic Cord for Chronic Scrotal Content Pain: A Novel Approach for Adult and Pediatric Patients. Urology 2019;131:144-9.

8. Heidenreich A, Olbert P, Engelmann UH. Management of Chronic Testalgia by Microsurgical Testicular Denervation. Eur Urol 2002;41:392-7.

9. Parekattil SJ, Gudeloglu A, Brahmbhatt JV, et al. Trifecta Nerve Complex: Potential Anatomical Basis for Microsurgical Denervation of the Spermatic Cord for Chronic Orchialgia. J Urol 2013;190:265-70.

10. Kavoussi PK. Validation of targeted microsurgical spermatic cord denervation: comparison of outcomes to traditional complete microsurgical spermatic cord denervation. Asian J Androl 2019;21:319-23.

11. Wagenlehner FME, van Till JWO, Magri V, et al. National Institutes of Health Chronic Prostatitis Symptom Index (NIH-CPSI) Symptom Evaluation in Multinational Cohorts of Patients with Chronic Prostatitis/Chronic Pelvic Pain Syndrome. Eur Urol 2013;63:953-9.

12. Shoskes DA, Calixte N, Tadros N, et al. Validation of the Chronic Orchialgia Symptom Index for Men With Chronic Orchialgia/Chronic Scrotal Contents Pain. Urology 2018;119:39-43.

13. Rotshenker S. Wallerian degeneration: the innate-immune response to traumatic nerve injury. J Neuroinflammation 2011;8:109.

14. Dubový P. Wallerian degeneration and peripheral nerve conditions for both axonal regeneration and neuropathic pain induction. Ann Anat 2011;193:267-75.

15. Üçeyler N, Sommer C. Wallerian degeneration and neuropathic pain. Drug Discov Today Dis Mech 2006;3:351-6.

16. Oka S, Shiraishi K, Matsuyama H. Microsurgical Anatomy of the Spermatic Cord and Spermatic Fascia: Distribution of Lymphatics, and Sensory and Autonomic Nerves. J Urol 2016;195:1841-7.

17. Shah JP, Danoff JV, Desai MJ, et al. Biochemicals associated with pain and inflammation are elevated in sites near to and remote from active myofascial trigger points. Arch Phys Med Rehabil 2008;89:16-23.

18. Moldwin RM, Fariello JY. Myofascial Trigger Points of the Pelvic Floor: Associations with Urological Pain Syndromes and Treatment Strategies Including Injection Therapy. Curr Urol Rep 2013;14:409-17.

19. Planken E, Voorham-van der Zalm PJ, Lycklama A Nijeholt AA, et al. Chronic testicular pain as a symptom of pelvic floor dysfunction. J Urol 2010;183:177-81.

20. Shoskes DA, Nickel JC, Kattan MW. Phenotypically directed multimodal therapy for chronic prostatitis/ chronic pelvic pain syndrome: a prospective study using UPOINT. Urology 2010;75:1249-53.

21. Farrell MR, Dugan SA, Levine LA. Physical therapy for chronic scrotal content pain with associated pelvic floor pain on digital rectal exam. Can J Urol 2016;23:8546-50.

22. Tan WP, Tsambarlis PN, Levine LA. Microdenervation of the spermatic cord for post-vasectomy pain syndrome. BJU Int 2018;121:667-73.

23. Larsen SM, Benson JS, Levine LA. Microdenervation of the spermatic cord for chronic scrotal content pain: single institution review analyzing success rate after prior attempts at surgical correction. J Urol 2013;189:554-8.
Cite this article as: Murthy PB, Parekh NV, Vij SC, Shoskes DA. Microscopic spermatic cord denervation for chronic orchialgia/ chronic scrotal content pain: operative outcomes and predictors of failure. Transl Androl Urol 2020;9(5):1931-1936. doi:10.21037/tau20-561 